\title{
Roles of Fas/Fasl, Bcl-2/Bax, and Caspase-8 in rat nonalcoholic fatty liver disease pathogenesis
}

\author{
C.P. Li ${ }^{1}$, J.H. Li ${ }^{2}$, S.Y. He ${ }^{1}$, P. $\mathrm{Li}^{1}$ and X.L. Zhong ${ }^{1}$ \\ ${ }^{1}$ Department of Gastroenterology, \\ The Affiliated Hospital of Luzhou Medical College, Luzhou, China \\ ${ }^{2}$ West China School of Medicine, Sichuan University, \\ Chengdu, China \\ Corresponding author: C.P. Li \\ E-mail: changpinglicn@126.com
}

Genet. Mol. Res. 13 (2): 3991-3999 (2014)

Received July 8, 2013

Accepted November 18, 2013

Published May 23, 2014

DOI http://dx.doi.org/10.4238/2014.May.23.10

\begin{abstract}
The aim of this study was to investigate the roles of Fas/ FasL, Bcl-2/Bax, and Caspase-8 mRNA expressions in nonalcoholic fatty liver disease (NAFLD). The apoptosis percentage was measured by flow cytometry, the immunohistochemical assay was performed for the determination of Fas, FasL, Bcl-2, and Bax expressions, and a real-time polymerase chain reaction (PCR) assay was performed to detect Caspase- 8 mRNA expression. Flow cytometry showed that the apoptosis percentage of the rat liver in the experimental group increased, which increased more obviously with the extension of modeling time. Immunohistochemistry showed that with increasing hepatic steatosis, Fas and FasL protein staining intensified and the number of positive cells increased; the number of positive cells for Bcl-2 and Bax gradually increased on the 4th, 8th, and 12th weeks in the experimental group, whereas the $\mathrm{Bcl}-2 / \mathrm{Bax}$ ratio decreased. The real-time PCR assay showed that Caspase- 8 mRNA expression increased with increasing hepatic steatosis and inflammation, exhibiting a progressively rising
\end{abstract}


trend. Hepatocyte apoptosis could promote NAFLD progression; Fas, FasL, and Caspase- 8 mRNA activation were important contributing factors to NAFLD. The upregulation of Bax and Bcl-2 expression might be one important mechanism of the apoptosis in NAFLD.

Key words: Nonalcoholic fatty liver disease; Apoptosis; Fas/FasL; Bcl-2/Bax; Caspase-8

\section{INTRODUCTION}

Nonalcoholic fatty liver disease (NAFLD) is a clinical pathological syndrome with the main feature of diffused hepatocyte macrosteatosis caused by liver damage factors other than alcohol or other clear factors (Björnsson and Angulo, 2007; Duvnjak et al., 2007). With the improvement of living standards and lifestyle changes, the incidences of obesity and diabetes have increased, and consequently, the NAFLD incidence has also increased in China, and has become the second largest liver disease after viral hepatitis (Farrell and Larter, 2006). NAFLD can progress to cirrhosis, liver failure, and hepatocellular carcinoma (Browning and Horton, 2004; Manco et al., 2008; Smith and Adams, 2011), resulting in serious public health consequences. Although the pathogenesis of NAFLD is still not very clear, there is evidence that liver cell apoptosis and related factors, Fas/FasL, Bcl-2/Bax protein, and Caspase-8, all play important roles in its genesis and development (Feldstein et al., 2003; Argo et al., 2009). In this study, an improved high-fat diet NAFLD rat model was established for the detection of the expressions of Fas, FasL, Bcl-2, Bax protein, and Caspase- 8 mRNA in liver tissue, in order to further clarify their roles and relationship to liver disease pathogenesis.

\section{MATERIAL AND METHODS}

\section{Grouping and modeling}

The rats were adaptively fed for 1 week, and then 40 rats were randomly divided into two groups according to a random table: 20 rats were assigned to the normal control group ( $\mathrm{C}$ group) and 20 rats were assigned to the NAFLD experimental group (F group). Each group was then subdivided into 2-, 4-, 8-, and 12-week groups, with 5 animals in each subgroup. The $\mathrm{C}$ group was fed a normal diet, while the $\mathrm{F}$ group was fed an improved high-fat diet (i.e., $2 \%$ cholesterol $+0.5 \%$ sodium cholate $+0.2 \%$ propylthiouracil pyrimidine $+5 \%$ sucrose $+10 \%$ lard $+82.3 \%$ basic forage), and the experimental animals had free to access food and water. This study was carried out in strict accordance with the recommendations in the Guide for the Care and Use of Laboratory Animals of the National Institutes of Health. The animal use protocol was reviewed and approved by the Institutional Animal Care and Use Committee (IACUC) of Luzhou Medical College.

\section{Sampling}

The rats were fasted for $12 \mathrm{~h}$ with free access to water the day before being killed. The next day, the body weight of each animal was determined, and they were intraperitoneally injected with $20 \mathrm{~g} / \mathrm{L} 1 \mathrm{~mL} / \mathrm{kg}$ sodium pentobarbital solution as anesthetic. The cardiac blood was 
obtained and the serum was extracted. Then, the liver was quickly removed and its wet weight was determined to calculate the liver index: liver wet weight/body weight x $100 \%$. Liver tissues were cut into $1.0 \mathrm{~cm}^{3}$ sections in the middle of the left liver lobe, immersed in $4 \%$ paraformaldehyde for fixation to prepare for biopsy, and the remaining liver tissue was stored at $-80^{\circ} \mathrm{C}$.

\section{Determination of blood lipids and liver functions}

An automatic biochemical analyzer was used for the detection of serum triglyceride (TG), total cholesterol (TC), alanine aminotransferase (ALT), and aspartate aminotransferase (AST) levels.

\section{Histopathological observations}

Fresh liver tissues were fixed in $4 \%$ paraformaldehyde, embedded in paraffin, and hematoxylin and eosin (HE)-stained, and were then observed under a microscope to determine the steatosis, inflammation, and fibrosis status.

\section{Flow cytometry Annexin V/PI method}

One gram fresh liver tissue was taken, placed in pre-chilled phosphate-buffered saline (PBS), and immediately cut into pieces. After several suspensions in PBS, tissues were washed and filtered with 400-mesh to obtain a liver cell suspension. Erythrocyte lysate was added for lysis for $10 \mathrm{~min}$ on ice, centrifuged at $1000 \mathrm{rpm}$ for $5 \mathrm{~min}$, and the supernatant was removed. Liver cells were resuspended in PBS, stained with Annexin-V and PI, and the cell number was determined.

\section{Immunohistochemistry}

Immunohistochemistry of the liver tissues was performed according to the steps outlined in the SP immunohistochemical staining kit and DAB chromogenic kit instructions. Positive cells were those in which brown staining appeared in the cell membrane or cytoplasm. The Image Plus 5.0 image analysis software was used to calculate the integration of optical density (IOD) value for the semi-quantitative analysis.

\section{Real-time polymerase chain reaction (PCR)}

According to the instructions of the TRIZOL reagent kit, total RNA was extracted from liver tissues, the RNA concentration and purity were detected, and the total mRNA extracted was reverse-transcribed to form cDNA. The number of amplification cycles (Ct value) was determined when the fluorescence intensity of each sample tube reached a specific threshold value, which were used to determine the amplification dynamics of each tube. The $\mathrm{Ct}$ value of the curve was compared to the logarithm value of the initial standard template to obtain a standard curve of the sample. The primer sequences were as follows: GAPDH upstream, 5'-TGACATCAAGAAGGTGGTGA-3', GAPDH downstream, 5'-TCATACCAGGAAATGA GCTT-3'; Caspase-8 gene upstream, 5'-GATGAGGCAGACTTTCTGCT-3', Caspase-8 gene 
downstream, 5'-CATAGTTCACGCCAGTCAGGAT-3'. A positive Caspase-8 product was a fragment of $163 \mathrm{bp}$, and the amplification conditions for Caspase- 8 amplification were: $94^{\circ} \mathrm{C}$ for $2 \mathrm{~min}, 94^{\circ} \mathrm{C}$ for $20 \mathrm{~s}, 54^{\circ} \mathrm{C}$ for $20 \mathrm{~s}$, and $74^{\circ} \mathrm{C}$ for $20 \mathrm{~s}$, for a total of 45 cycles.

\section{Statistical analysis}

Data were statistically analyzed using the SPSS13.0 software. Measurement data are reported as means $\pm \mathrm{SD}$. Within-group comparisons of the $\mathrm{C}$ and $\mathrm{F}$ groups were conducted using the two-sample Student's $t$-test; between-group comparisons of the $\mathrm{C}$ and $\mathrm{F}$ groups were conducted with analysis of variance. Count data were evaluated with Fisher's exact test, and correlations were evaluated with linear correlation analysis. $\mathrm{P}<0.05$ was considered to be statistically significant.

\section{RESULTS}

\section{Serum lipids and liver function}

With increasing modeling time, serum lipid levels and liver function indexes in each F subgroup increased progressively; ALT showed the most obvious increase, which differed significantly at each time point, in both the within-group comparison and when compared to the $\mathrm{C}$ group (all $\mathrm{P}<0.01$ ). At 4 weeks, the $\mathrm{TG}$ levels in the $\mathrm{F}$ group increased significantly compared to those in group $\mathrm{C}$ and to the 2 -week value in the $\mathrm{F}$ group $(\mathrm{P}<0.01)$. The TC values of the $\mathrm{F}$ group differed significantly from those of the $\mathrm{C}$ group at each time point $(\mathrm{P}<$ $0.05)$. The AST levels were significantly different between the $\mathrm{F}$ and $\mathrm{C}$ groups $(\mathrm{P}<0.01)$. The within-F group comparison showed that the AST value at 4 weeks differed significantly from that at 2 weeks $(\mathrm{P}<0.01)$, whereas there were no significant differences among the 4-, 8-, and 12-week subgroups of the F group.

\section{Histological pathology}

The HE staining results showed no evidence of abnormalities in the rat livers of the $\mathrm{C}$ group (Figure 1A), whereas the liver inflammation scores of the $\mathrm{F}$ group were higher than those of the $\mathrm{C}$ group at each time point $(\mathrm{P}<0.05)$. The within-group comparison of the $\mathrm{F}$ group showed significant differences between the 4-week subgroup (Figure 1B) and the 2-week subgroup (Figure 1C) $(\mathrm{P}<0.01)$, and the 12-week (Figure1D) subgroup also differed significantly from the 8-week subgroup (Figure 1E) $(\mathrm{P}<0.05)$ (Figure 1).

\section{Apoptosis percentage}

The 2-week subgroup (Figure 2A) of the $\mathrm{F}$ group showed a slightly increased apoptosis percentage compared to the $\mathrm{C}$ group (Figure 2B), but the difference was not significant. The 4-week (Figure 2C), 8-week (Figure 2D), and 12-week subgroups (Figure 2E) of the F group all showed significantly increased hepatocyte apoptosis percentages compared to the same time point subgroups of the $\mathrm{C}$ group (Figure $2 \mathrm{~B}$ ) (all $\mathrm{P}<0.01)$. The within-group comparison of the $\mathrm{F}$ group showed a significant increase in the liver cell apoptosis percentage over time $(\mathrm{P}<0.01)$, whereas there were no significant differences in apoptosis among the $\mathrm{C}$ group subgroups (Figure 2B). 

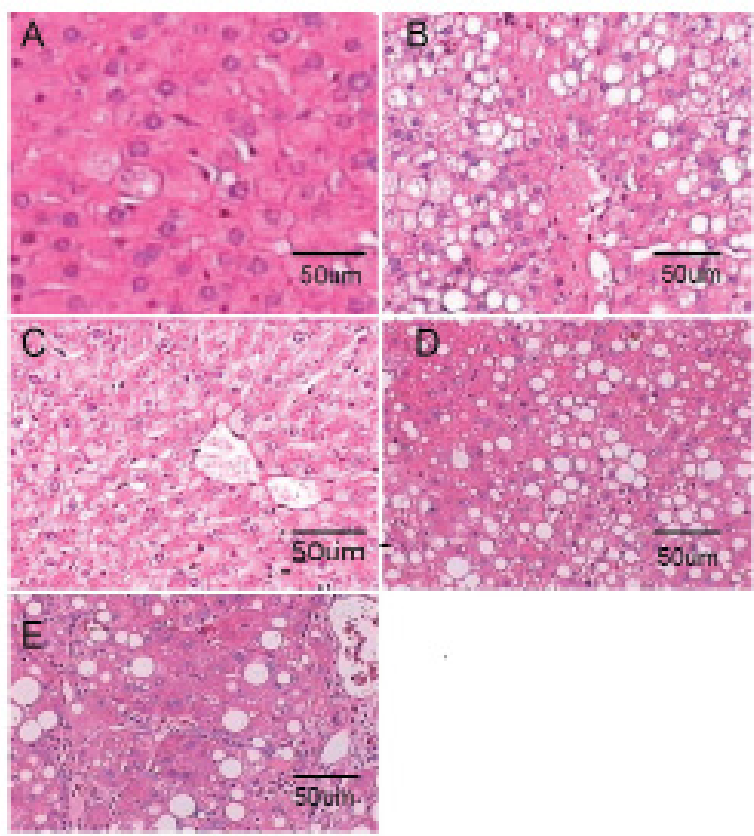

Figure 1. Histopathology of rat liver in each group (HE 400X). A. C group. B. F group, 2 weeks. C. F group, 4 weeks. D. F group, 8 weeks. E. F group, 12 weeks.
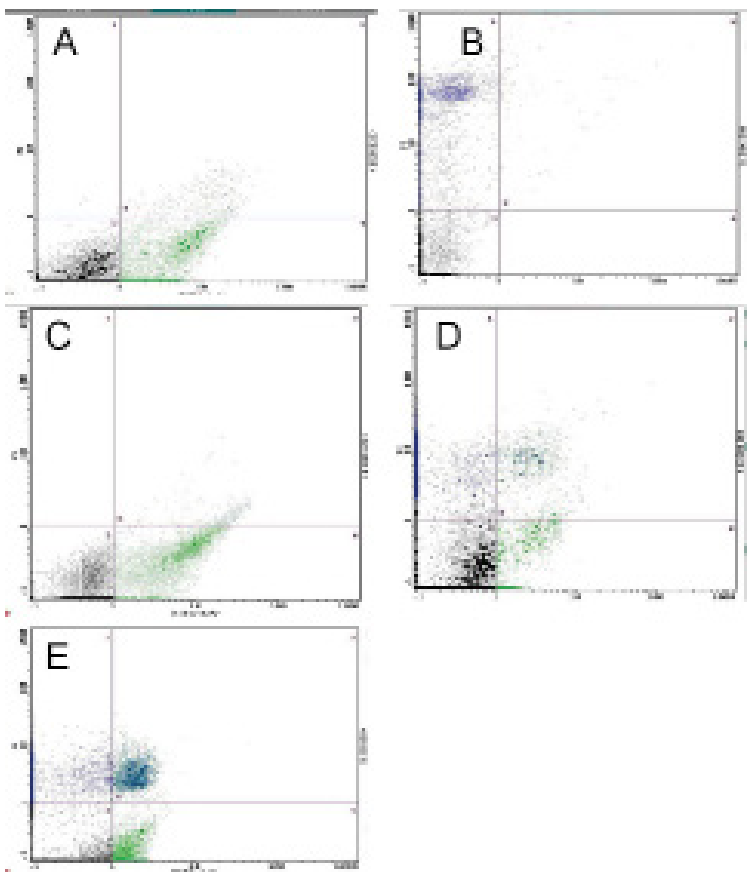

Figure 2. Hepatocyte apoptosis situation by flow cytometry. A. C group. B. F group, 2 weeks. C. F group, 4 weeks. D. F group, 8 weeks. E. F group, 12 weeks. 


\section{Fas and FasL protein expressions}

There were no significant differences in Fas and FasL expressions among the C subgroups, whereas the Fas and FasL levels of the $\mathrm{F}$ group gradually increased over time, with significant differences when compared with the $C$ group $(P<0.01)$. The 12 -week subgroup of the F group showed a significant increase compared to the 2- and 4-week subgroups, while there was only a small difference between the 8-week subgroup and the 12-week subgroup of the F group (Table 1).

Table 1. Fas/FasL, Bcl-2/Bax and Caspase-8 mRNA expression in rat liver tissue of two groups (means \pm SD,
IOD value).
\begin{tabular}{llccccccc}
\hline Group & & N & Fas & FasL & Bcl-2 & Bax & Bcl-2/Bax & Caspase-8mRNA $\left(2^{-\Delta \Delta C t}\right)$ \\
\hline C group & 2 weeks & 5 & $5.43 \pm 0.97^{\mathrm{b}}$ & $6.10 \pm 0.48$ & $3.87 \pm 0.76$ & $7.98 \pm 0.34$ & $0.48 \pm 0.17$ & $1.03 \pm 0.24^{\mathrm{b}}$ \\
& 4 weeks & 5 & $4.68 \pm 1.24^{\mathrm{b}}$ & $6.35 \pm 0.58$ & $4.13 \pm 0.97^{\mathrm{b}}$ & $8.70 \pm 0.66^{\mathrm{b}}$ & $0.46 \pm 0.16$ & $1.10 \pm 0.36^{\mathrm{b}}$ \\
& 8 weeks & 5 & $6.70 \pm 1.82^{\mathrm{b}}$ & $8.25 \pm 1.59^{\mathrm{b}}$ & $5.63 \pm 1.31^{\mathrm{b}}$ & $8.70 \pm 0.74^{\mathrm{b}}$ & $0.63 \pm 0.18$ & $1.87 \pm 0.56^{\mathrm{b}}$ \\
& 12 weeks & 5 & $4.51 \pm 2.26^{\mathrm{b}}$ & $9.43 \pm 1.75^{\mathrm{b}}$ & $6.42 \pm 2.25$ & $29.45 \pm 1.45^{\mathrm{b}}$ & $0.67 \pm 0.21$ & $1.92 \pm 0.65^{\mathrm{a}}$ \\
F group & 2 weeks & 5 & $9.90 \pm 3.93$ & $8.87 \pm 0.76^{\mathrm{d}}$ & $4.74 \pm 0.37$ & $9.53 \pm 1.51^{\mathrm{d}}$ & $0.48 \pm 0.24$ & $2.54 \pm 0.32^{\mathrm{d}}$ \\
& 4 weeks & 5 & $11.90 \pm 5.49$ & $17.00 \pm 1.34^{\mathrm{f}}$ & $6.18 \pm 1.58$ & $24.60 \pm 2.81^{\mathrm{f}}$ & $0.29 \pm 0.14$ & $5.46 \pm 0.93^{\mathrm{f}}$ \\
& 8 weeks & 5 & $14.42 \pm 6.31$ & $30.10 \pm 7.31$ & $8.94 \pm 1.56$ & $37.90 \pm 1.69^{\mathrm{h}}$ & $0.21 \pm 0.12$ & $8.15 \pm 1.48^{\mathrm{h}}$ \\
& 12 weeks & 5 & $18.43 \pm 5.47$ & $39.57 \pm 6.43$ & $9.16 \pm 3.42$ & $46.00 \pm 3.22$ & $0.18 \pm 0.08$ & $17.40 \pm 1.97$ \\
\hline
\end{tabular}

${ }^{\mathrm{a}} \mathrm{P}<0.01$, vs $\mathrm{F}$ group, 12 weeks; ${ }^{\mathrm{b}} \mathrm{P}<0.01$ vs each time point of $\mathrm{F}$ group; ${ }^{\mathrm{d}} \mathrm{P}<0.01$ vs $\mathrm{F}$ group 4 weeks; ${ }^{\mathrm{f}} \mathrm{P}<0.01 v s$ F group, 8 weeks; ${ }^{\mathrm{P}}<0.01$ vs $\mathrm{F}$ group, 12 weeks.

\section{Bcl-2/Bax protein expression}

Bcl-2 and Bax protein expressions in the $\mathrm{F}$ group increased over time, with a statistically significant difference when compared to the $\mathrm{C}$ group $(\mathrm{P}<0.01)$. The $\mathrm{Bcl}-2 / \mathrm{Bax}$ ratio of the $\mathrm{F}$ group gradually decreased with fatty liver progress, with the most obvious effects observed in the 8-week and 12-week subgroups (Table 1).

\section{Caspase-8 mRNA relative expression}

The relative expression levels of Caspase- 8 mRNA in the F group increased progressively with increasing fatty liver severity, and the Caspase- 8 mRNA expression levels of the F group were also significantly higher than those in the $\mathrm{C}$ group at each time point (all $\mathrm{P}<0.01$ ); there were significant differences among the $\mathrm{F}$ subgroups (all $\mathrm{P}<0.01$; Table 1 ).

\section{DISCUSSION}

Hepatocyte apoptosis is a common pathological manifestation of various liver diseases, and in recent years, great progress has been made with respect to understanding its role in the occurrence and development of NAFLD. Studies have revealed that hepatocyte apoptosis plays a vital role in the progress of NAFLD to nonalcoholic steatohepatitis (NASH), liver fibrosis, and even cirrhosis and liver cancer (Schattenberg et al., 2006; Wieckowska et al., 2006). In this study, we successfully established an NAFLD rat model, as evidenced by elevated serum ALT, AST, TC, and TG levels, and liver histology showed liver steatosis, ne- 
crosis, and inflammatory cell infiltration or associations with liver fibrosis, and the hepatocyte apoptosis increased significantly with the extension of modeling time.

The occurrence of hepatocyte apoptosis is mainly regulated by death receptor signal transduction pathways, mitochondria signal pathways, and the endoplasmic reticulum signaling pathway (Harper et al., 2003; Canbay et al., 2004). Fas, which is involved in the death receptor signal transduction pathway, is a transmembrane protein belonging to the superfamily of tumor necrosis factor receptors, and plays a role in the signal transduction of apoptosis (Myśliwiec et al., 2006). The combination of Fas and FasL could initiate transduction of the lethal apoptotic signal, thereby promoting cell apoptosis. High expression of the Fas protein could bind soluble FasL (Contassot et al., 2007), leading to the flotation of Fas-associated death domain protein (FADD), forming the death-inducing signaling complex (DISC), activating procaspase-8, causing the release of the upstream Caspase-8 (Iwase et al., 2006; WescheSoldato et al., 2007), subsequently activating Caspase-3, Caspase-6, and Caspase-7, which induces the chain reaction of the Caspase family (Yin and Ding, 2003; Gao et al., 2007), ultimately acting on structural proteins of cells, leading to cell apoptosis. Caspase- 8 is a key enzyme acting in the upstream apoptosis pathway, and also acts as an important apoptosis initiation factor by activating almost all of the downstream Caspases in the apoptotic cascade (Fan et al., 2005). The experimental results showed that the expression of Fas, FasL, and Caspase- 8 mRNA in the F group increased with NAFLD hepatic steatosis; the expression level increased gradually showing a positive correlation with NAFLD liver steatosis levels and the hepatic apoptosis percentage, suggesting that the incidence and development of liver disease are related to the upregulation of Fas, FasL, and Caspase-8. One possible mechanism might be the following: the high-fat diet caused hepatic steatosis, fat decomposition generated free fatty acids (FFA), and consequently, the lipid toxicity of FFA might have induced apoptosis of fatty liver cells and oxidative stress, thereby producing large amounts of reactive oxygen species (ROS). The generation of ROS could then activate the Fas/FasL system, further leading to structural proteins of the Fas lethal zone to recruit downstream Caspase family members (Häcker and Paschen, 2007), promoting the formation of the protease cascade, thus leading to liver cells apoptosis.

In the mitochondrial-regulating apoptosis system, Bcl-2 and its family members together constitute a very complex interaction network regulating apoptosis (Begriche et al., 2006), among which Bcl-2 and Bax are the most important representatives. In the NAFLD formation process, fat degeneration and oxidative stress could increase Bax synthesis and move outside of the mitochondrial membrane, thereby changing the configuration and forming homodimers, inducing the opening of the mitochondrial PT pore and the release of cytochrome $\mathrm{C}$, which would further induce apoptosis. Bcl-2 is mainly distributed in the outer mitochondrial membrane; its homodimer would suppress the opening of the PT pore, and its combination with Bax could prevent Bax's conformational change and thus play a role in antiapoptosis (Pastorino et al., 2003; Akcali et al., 2004). Therefore, Bcl-2 inhibits apoptosis while Bax promotes apoptosis, and the ratio of the $\mathrm{Bcl}-2 / \mathrm{Bax}$ protein complex is directly related to the occurrence of apoptosis. Indeed, some scholars have even named the Bcl-2/Bax ratio as the "apoptosis switch". When the Bax protein is dominant, apoptosis occurs, whereas when the Bcl-2 protein is dominant, the cell survives (Ramalho et al., 2006). The present experiment showed that the expressions of the Bcl-2 protein and the Bax protein in NAFLD liver cells increased compared with the normal control group. However, in the process of liver fat degeneration and inflammatory change development, the expression of the Bax protein increased 
significantly relative to $\mathrm{Bcl}-2$ protein expression, thus progressively reducing the $\mathrm{Bcl}-2 / \mathrm{Bax}$ ratio while significantly increasing the apoptosis percentage of liver cells. In addition, the Bcl2/Bax ratio was negatively correlated with hepatocyte apoptosis; thus, it could be speculated that the imbalance of Bcl-2 and Bax might be one important factor contributing to the increase observed in hepatocyte apoptosis.

In summary, the present study provides an in-depth analysis of hepatocyte apoptosis in NAFLD through evaluation of the dynamic hepatocyte apoptosis percentage and the expressions of liver Fas/FasL, Bcl-2/Bax, and Caspase-8. Because the apoptosis molecular factors Fas/FasL, Bcl-2/Bax, and Caspase-8 are closely related with hepatocyte apoptosis, NAFLD pathogenesis could be investigated, thus providing a strong theoretical basis of apoptosis that can be used for NAFLD diagnosis and treatment, providing a new approach for the clinical diagnosis, treatment, and prognosis of NAFLD.

\section{ACKNOWLEDGMENTS}

Research supported by the Luzhou Municipal Science and Technology Bureau Foundation project, \#2011-I-S39 (2/6).

\section{REFERENCES}

Akcali KC, Dalgic A, Ucar A, Haj KB, et al. (2004). Expression of bcl-2 gene family during resection induced liver regeneration: comparison between hepatectomized and sham groups. World J. Gastroenterol. 10: 279-283.

Argo CK, Northup PG, Al-Osaimi AM and Caldwell SH (2009). Systematic review of risk factors for fibrosis progression in non-alcoholic steatohepatitis. J. Hepatol. 51: 371-379.

Begriche K, Igoudjil A, Pessayre D and Fromenty B (2006). Mitochondrial dysfunction in NASH: causes, consequences and possible means to prevent it. Mitochondrion 6: 1-28.

Björnsson E and Angulo P (2007). Non-alcoholic fatty liver disease. Scand. J. Gastroenterol. 42: 1023-1030.

Browning JD and Horton JD (2004). Molecular mediators of hepatic steatosis and liver injury. J. Clin. Invest. 114: $147-152$.

Canbay A, Friedman S and Gores GJ (2004). Apoptosis: the nexus of liver injury and fibrosis. Hepatology 39: 273-278.

Contassot E, Gaide O and French LE (2007). Death receptors and apoptosis. Dermatol. Clin. 25: 487-501.

Duvnjak M, Lerotic I, Barsic N, Tomasic V, et al. (2007). Pathogenesis and management issues for non-alcoholic fatty liver disease. World J. Gastroenterol. 13: 4539-4550.

Fan TJ, Han LH, Cong RS and Liang J (2005). Caspase family proteases and apoptosis. Acta Biochim. Biophys Sin. 37 : 719-727.

Farrell GC and Larter CZ (2006). Nonalcoholic fatty liver disease: from steatosis to cirrhosis. Hepatology 43: S99-S112.

Feldstein AE, Canbay A, Angulo P, Taniai M, et al. (2003). Hepatocyte apoptosis and fas expression are prominent features of human nonalcoholic steatohepatitis. Gastroenterology 125: 437-443.

Gao Z, Tian Y, Wang J, Yin Q, et al. (2007). A dimeric Smac/diablo peptide directly relieves caspase-3 inhibition by XIAP. Dynamic and cooperative regulation of XIAP by Smac/Diablo. J. Biol. Chem. 282: 30718-30727.

Häcker G and Paschen SA (2007). Therapeutic targets in the mitochondrial apoptotic pathway. Expert Opin. Ther. Targets 11: 515-526.

Harper N, Hughes M, MacFarlane M and Cohen GM (2003). Fas-associated death domain protein and caspase-8 are not recruited to the tumor necrosis factor receptor 1 signaling complex during tumor necrosis factor-induced apoptosis. J. Biol. Chem. 278: 25534-25541.

Iwase M, Kondo G, Watanabe H, Takaoka S, et al. (2006). Regulation of Fas-mediated apoptosis in neutrophils after surgery-induced acute inflammation. J. Surg. Res. 134: 114-123.

Manco M, Bottazzo G, DeVito R, Marcellini M, et al. (2008). Nonalcoholic fatty liver disease in children. J. Am. Coll. Nutr. 27: 667-676.

Myśliwiec J, Okota M, Nikolajuk A and Górska M (2006). Soluble Fas, Fas ligand and Bcl-2 in autoimmune thyroid diseases: relation to humoral immune response markers. Adv. Med. Sci. 51: 119-122. 
Pastorino JG, Shulga N and Hoek JB (2003). TNF-alpha-induced cell death in ethanol-exposed cells depends on p38 MAPK signaling but is independent of Bid and caspase-8. Am. J. Physiol. Gastrointest. Liver Physiol. 285: G503-G516.

Ramalho RM, Cortez-Pinto H, Castro RE, Solá S, et al. (2006). Apoptosis and Bcl-2 expression in the livers of patients with steatohepatitis. Eur. J. Gastroenterol. Hepatol. 18: 21-29.

Schattenberg JM, Galle PR and Schuchmann M (2006). Apoptosis in liver disease. Liver Int. 26: 904-911.

Smith BW and Adams LA (2011). Non-alcoholic fatty liver disease. Crit. Rev. Clin. Lab. Sci. 48: 97-113.

Wesche-Soldato DE, Chung CS, Gregory SH, Salazar-Mather TP, et al. (2007). CD8+ T cells promote inflammation and apoptosis in the liver after sepsis: role of Fas-FasL. Am. J. Pathol. 171: 87-96.

Wieckowska A, Zein NN, Yerian LM, Lopez AR, et al. (2006). In vivo assessment of liver cell apoptosis as a novel biomarker of disease severity in nonalcoholic fatty liver disease. Hepatology 44: 27-33.

Yin XM and Ding WX (2003). Death receptor activation-induced hepatocyte apoptosis and liver injury. Curr. Mol. Med. 3: 491-508. 